\title{
Estrategias para la enseñanza del lenguaje académico. Dominio desde la instrucción de las destrezas orales
}

\author{
ESTHER CORTÉS BUENO \\ Universidad de Córdoba \\ cu9cobue@uco.es
}

Resumen: La presencia cada vez más numerosa de estudiantes de movilidad procedentes de distintos países comunitarios y extracomunitarios en las aulas de nuestras universidades hace necesario habilitar un espacio curricular para ellos. No hay que olvidar que su presencia es parte de los objetivos marcados por el proyecto del Espacio Europeo de Enseñanza Superior (EEES); y una correcta integración de este tipo de alumnado respondería a las aspiraciones de internacionalización de la Universidad. La pretensión de esta propuesta es exponer el mecanismo de los cursos en los que a partir de la instrucción en las destrezas orales se establece la base para profundizar en el aprendizaje de la expresión escrita en distintos géneros textuales académicos. Se trabaja sobre una rica tipología de géneros textuales, con enfoques que fomentan el uso real de la lengua.

Palabras clave: Enseñanza universitaria, Espacio Europeo de Educación Superior, géneros textuales, español lengua extranjera, currículo integrado AICLE.

\begin{abstract}
The ever increasing number of students studying in our university classrooms proceding from within and outwith the European community, create the need for a special curricular programme for these students. It should not be forgotten that their presence is part of the objectives of the project of the European Space of Higher Education (EHEA): a correct integration of this students would respond to the aspirations of internationalization of the University. The aim of this proposal is to explain the mechanism of courses whereby the teaching of oral skills form the foundations for a deeper learning of written skills in different academic contexts, working on a rich variety of tipologies of textual genre, with emphasis on the true use of the language.
\end{abstract}

Key words: University learning, European Higher Education, academic genres, Spanish foreign language, integrated curriculum. 
En este artículo exponemos la situación actual, dentro del ámbito universitario, en la que se encuentran los estudiantes extranjeros de movilidad durante su estancia en España y proponemos un plan de actuación para responder a las nuevas necesidades. Nuestro estudio se enmarca dentro del proyecto del Espacio Europeo de Enseñanza Superior (EEES) y, al mismo tiempo, responde a las aspiraciones de internacionalización de las universidades europeas. Atendiendo a la política del EEES, estos estudiantes visitantes han de ser considerados como parte integrante de la comunidad universitaria, con todo lo que esto conlleva: derechos y obligaciones. Entre sus obligaciones, han de integrarse en el aula universitaria bajo los criterios de evaluación general (en cuanto a los contenidos) y las mismas exigencias lingüísticas que tienen sus compañeros locales, aunque la realidad es que el desequilibrio lingüístico entre los dos tipos de aprendices, los locales y los extranjeros, dificulta la participación de estos últimos. Una formación específica de español como lengua extranjera para estos últimos podría ofrecerse como un derecho y les permitiría mejorar su dominio comunicativo y, de forma específica académico, aumentando su aprovechamiento en la universidad.

Este EEES puede considerarse la materialización de parte de una política europea que se inició una vez terminada la II Guerra Mundial, no muchos años después de la I Guerra Mundial, en un espacio devastado, hundido en una crisis económica sin precedentes, con una población activa mermada y una Europa disgregada.

Los primeros pasos que impulsaron la Comunidad Económica Europea (CEE), a partir de su creación en 1958, se encaminaron a fortalecer la cooperación económica entre sus países miembros con el propósito de reestablecer la situación económica e industrial y evitar nuevos conflictos raciales y, en definitiva, bélicos. Su política consistía en generar unas condiciones que favorecieran una unión duradera con el convencimiento de que, a medida que se estrechara la cooperación económica y se eliminaran las fronteras, se iría creando una conciencia europea entre sus ciudadanos. El conocimiento cultural y lingüístico haría posible el paso de unión únicamente económica a una unión que beneficiase a todos los órdenes, convertida a partir de 1993 en la Unión Europea.

En este contexto y en el empeño de alcanzar una Europa plurilingüe se gestó la política lingüística europea financiada y promovida a través del Consejo de Europa y la Comisión. Con esto podría lograrse igualmente una conciencia europea entre sus habitantes, que facilitaría la movilidad de los trabajadores y reduciría los conflictos raciales, como declara el Consejo de Europa en su fundación:

La finalidad del Consejo de Europa consiste en realizar una unión más estrecha entre sus miembros para salvaguardar y promover los ideales y los principios que constituyen su patrimonio común y favorecer su progreso económico

Consejo de Europa (1949: artículo 1)

Esta política lingüística (COM, 2008) financiaba la inclusión en los distintos países miembros de la enseñanza y aprendizaje de tres lenguas, a saber: la lengua materna, la lengua franca o de comunicación internacional (el inglés básicamente) y la lengua adoptiva materna. El Consejo publicó una serie de recomendaciones encaminadas a cumplir este objetivo ( $\mathrm{n}^{\circ} 18$ de CE, 1982), mediante una Educación Permanente de idiomas a lo largo de la vida escolar y profesional e incentivando la movilidad entre estudiantes y profesorado:

\section{Educación obligatoria}




\section{Educación Profesional \\ Educación Universitaria \\ Durante la vida Laboral}

Esta política ha estado unida desde el inicio a una serie de ayudas económicas y administrativas como becas (Erasmus, etc.) o la unificación de las titulaciones entre universidades gracias a medidas administrativas (Declaración de Bolonia) y a la unificación de los criterios evaluadores.

Fruto de estas recomendaciones son los planes lingüísticos de las universidades de la Unión Europea (UE) que acogen estudiantes extranjeros. Estos tienen el derecho de recibir educación adecuada a las exigencias universitarias y la universidad la obligación de proporcionar los medios, respondiendo a la idea de universalidad que fundamenta la Universidad. En este artículo tomamos como ejemplo el caso de la Universidad de Córdoba (España) debido a que, siendo el lugar en el que impartimos docencia, es también una de las que están experimentando este fenómeno.

El Plan de Fomento de Plurilingüismo (2014-2017) de la Universidad de Córdoba aprobado por su Consejo de Gobierno en Sesión ordinaria de 18 de diciembre de 2014 (BOUCO, 2014) pretende incentivar el plurilingüismo estudiantil y del profesorado. Este Plan se enmarca dentro de las pretensiones de internacionalización de las universidades (Pastor Cesteros, 2014: 22), mediante la oferta de titulaciones bilingües y otras medidas. Para ello, hubo de poner en marcha una ruta de formación lingüística para la comunidad universitaria ligada a unas exigencias lingüísticas que el alumnado y profesorado debían acreditar dentro de unos plazos marcados (UCO, 2014: artículo 2 del capítulo I).

Esta situación ha creado unas necesidades urgentes, movilizando a toda la comunidad universitaria para dar una respuesta efectiva, como ha sido la oferta de cursos o medidas dirigidas a la formación del alumnado y profesorado, $\mathrm{y}$ otras a modo de incentivos (prioridad en los programas de movilidad) y compensaciones. Una de las medidas más eficaces para su canalización ha sido la creación de la figura de un coordinador de política lingüística, dependiente directamente del Rector, que coordina el trabajo del profesorado según un aprendizaje integrado de contenidos de áreas no lingüísticas (ANL) y lengua, como AICLE.

Sin embargo, esta política está destinada al profesorado y alumnado locales, sin contar con los estudiantes extranjeros participantes de los programas de movilidad. En nuestra opinión, este es un paso necesario para hablar de internacionalización de la universidad (Pastor Cesteros, 2014: 22). Por otra parte, no debemos olvidar las recomendaciones comunitarias según las cuales la Universidad ha de integrar a los estudiantes extranjeros durante el periodo en el que estos formen parte de su comunidad aunque sea temporalmente (CE, 2001: 168; Gutiérrez Rivilla, 2004: 620).

Podríamos pensar que, como los estudiantes extranjeros que acoge la universidad han recibido formación lingüística desde la infancia y de manera permanente, estos disfrutan de un alto nivel de lengua española. Por el contrario, la mayoría no cuenta con un mínimo dominio para enfrentarse a las tareas universitarias. La lengua inglesa es la lengua franca 
y el español en la UE ocupa un cuarto lugar en el estudio escolar (Eurostat, 2015) ${ }^{1}$ durante la enseñanza secundaria, sin llegar a alcanzar normalmente el nivel de lengua adoptiva.

Por otra parte, la Universidad de Córdoba, como la mayoría de las universidades españolas, no tiene un requisito mínimo de competencia lingüística para este alumnado (Pastor Cesteros, 2014: 19). Esto permite recibir a un mayor número de estudiantes pero «como contrapartida» dificulta su integración y participación en la universidad. El Instituto Cervantes (IC) establece el nivel B2 como el mínimo adecuado y competente para los contenidos académicos y de especialidad, por ser el que capacita «al estudiante para comprender textos orales y escritos complejos aunque versen sobre temas abstractos, se presenten en diversas variedades del español o tengan un carácter técnico, principalmente si trata sobre áreas de conocimiento especializado» (IC, 2013: 6). De no contar con una competencia adecuada, el alumno no puede desarrollar su actividad académica, y su estancia en la universidad de acogida no servirá para cumplir los objetivos de la política europea respecto al EEES. Considerando que la lengua, desde su concepción comunicativa, forma parte de todas las asignaturas (Pavón Vázquez, 2006: 420; Fernández López, 2011: 216), sería aconsejable convertir la lengua de instrucción en el lazo de unión entre las distintas materias (Gómez Camacho, 2013). Partiendo de esta filosofía, proponemos un plan de formación desde la coordinación del profesorado de distintas áreas de conocimiento y el de lengua instrumental, donde todas las partes tomen un papel activo en la adecuación lingüística del estudiante extranjero (CE, 2001: 168) para un aprovechamiento e integración acorde a los objetivos comunitarios (Méndez y Pavón, 2012: 584). Por todo ello, resulta básico la formación y adecuación lingüística del alumnado visitante de movilidad durante su estancia en la universidad de acogida.

A lo largo de los últimos años, en la Universidad de Córdoba desde su Servicio de Lenguas Modernas (UCOidiomas) se ha trabajado para ofrecer al estudiante extranjero una formación que responda a sus necesidades. Después de años de experiencia hemos comprendido que la coordinación entre las distintas instituciones universitarias y el profesorado participante es imprescindible para una actuación eficiente. El aprendizaje de una lengua extranjera no termina en el aula de idiomas sino que ha de extenderse al resto de la actividad académica e incluso alcanzar la vida personal y social.

En este sentido, el enfoque de un Aprendizaje integrado de Contenidos y lengua (AICLE) permite que el estudiante extranjero aprehenda los conocimientos a través de la nueva lengua. La universidad se convierte en una plataforma de aprendizaje no sólo de las áreas de contenidos de especialidad. Gracias a este enfoque integrado, la lengua se aprende a través de la realidad e intereses del usuario. Al mismo tiempo, la lengua (su conocimiento y dominio) se convierte en un instrumento cognitivo con el que afrontar el resto de las asignaturas.

Esta propuesta requiere de la intervención institucional que facilite los medios y fomente la participación y el compromiso del profesorado que recibirá formación específica y apropiada.

\section{Propuesta de integración de la enseñanza del español en los planes lingüísticos}

\footnotetext{
${ }^{1}$ Los datos a los que nos referimos pueden consultarse en http://ec.europa.eu/eurostat/statisticsexplained/index.php/Foreign_language_learning_statistics
} 
Para este plan se proponen las mismas líneas de actuación básicas del Plan de Política Lingüística (BOUCO, 2014) promovido por el área de lenguas extranjeras de la Universidad de Córdoba en el Proyecto de mejora de la calidad docente (UCO, 2011). De esta forma, contaría con el respaldo institucional y logístico (Marsh, Pavón y Frigols, 2013) sobre el que activar un Programa de Adecuación y Mejora Lingüística, cuyo conocimiento habría de hacerse llegar a los interesados mediante la publicidad necesaria facilitada por la propia Universidad (Pastor Cesteros, 2014: 23) y la implicación de todos los responsables educativos, bajo la figura de un coordinador (Trujillo, 2010; Méndez y Pavón, 2012: 575; Pavón Vázquez, 2014: 117).

El estudiante extranjero está en un contexto de inmersión donde la lengua española es lengua instrumental y vehicular, materna para los estudiantes locales y extranjera para los estudiantes de movilidad por lo que existe un déficit lingüístico por parte de estos últimos, generando unas condiciones de desigualdad. Teniendo en cuenta esta situación, la primera medida sería considerar la lengua española como lengua extranjera de forma que sea posible participar en el Plan de Fomento de Plurilingüismo (BOUCO, 2014) y aprovechar las ventajas que reportaría un apoyo logístico institucional.

Una vez alcanzado este primer paso, se organizarían cursos específicos de español como lengua extranjera (ELE) instrumental, ajustando el currículo a las necesidades académicas del estudiante. De la misma forma, el profesorado universitario, tras una formación idónea, incorporaría este enfoque en aquellas clases donde participara este tipo de estudiante extranjero. La coordinación y el apoyo entre el profesorado permitirá activar un aprendizaje global y eficiente.

No obstante, teniendo en cuenta la complejidad de esta implicación del profesorado, en su defecto, los cursos específicos de ELE pueden concebirse como cursos de apoyo y adecuación lingüística a las restantes materias universitarias, siempre que el profesorado universitario esté abierto a ofrecer la información necesaria para la elaboración de planes curriculares. Si bien, de esta forma limitada, no se trataría de AICLE, es posible aprovechar la celebración de cursos de lengua específicos para las necesidades de un estudiante universitario.

Además, mantener el contacto con el profesorado de ANL, no sólo reporta la información necesaria para la elaboración curricular sino que fomenta la sensibilización del profesorado ante este tipo de alumnos y, presumiblemente, repercute en los métodos de estudio y de trabajo.

\section{Metodología de los cursos de español}

Estos cursos de español han de tener un enfoque específico con el que responder a unos objetivos también específicos. Proponemos el enfoque por tareas donde el género textual sea la unidad de trabajo (Martínez-Atienza y Perea, 2015). El estudio de los géneros textuales se aborda desde las cuatro destrezas escritas y orales, organizando los contenidos por unidades didácticas.

La naturaleza de estos cursos de lengua viene marcada por sus objetivos y estaría condicionada a factores variables (tipo de alumnado: necesidades lingüísticas relacionadas con sus estudios, nivel de competencia lingüística, lengua materna, etc.) que estarán presentes a la hora de elaborar el plan curricular. Teniendo en cuenta estas variables, se especifican los contenidos, estrategias, tipos y criterios de evaluación. 
La lengua ha de ser tratada en un nivel superior de géneros textuales (Lorenzo Berguillos, 2013), centrándose cuando los grupos sean homogéneos en sus materias de conocimiento (Bassols y Torrent, 1997; Martínez-Atienza y Perea, 2015; Pastor Cesteros, 2014), pero en caso de carecer de un alumnado homogéneo, la tipología textual será el eje que vertebre cada unidad. En la enseñanza de lenguas extranjeras (LE), los géneros discursivos se relacionan con la enseñanza para fines específicos. Sin embargo, deberían emplearse en cualquier nivel. El género discursivo se define según parámetros externos y contextuales (propósito comunicativo, estatus del emisor y receptor, tipo y modo de interacción) y se diferencian de los tipos de texto (definidos por características internas, estructurales y gramaticales), al ser un repertorio abierto a los cambios, adaptable a distintos niveles. Es tarea del profesor elegir el tipo de texto atendiendo al nivel de dominio y objetivos. Si se pretende alcanzar competencia comunicativa, el objetivo en la enseñanza es desarrollar la competencia discursiva (CE, 2001: 120) que se lleva a cabo reconociendo y trabajando los rasgos lingüísticos y discursivos que caracterizan cada tipo textual y no mediante un dominio en la competencia gramatical, en unidades menores al texto, como viene siendo habitual (Martín Peris, 2010).

Estructurar el currículo en función de géneros discursivos permite secuenciar los contenidos en unidades didácticas, donde se combinan para un mismo género distintos canales (oral y escrito), niveles de competencia y registros (familiar, culto, académico, etc.), manteniendo coherencia temática y metodológica. Un mismo género textual se emplea en numerosas circunstancias por lo que puede relacionarse con más de una tarea. El enfoque por tareas (CE, 2001: capítulo 7) resulta adecuado para alcanzar unos objetivos (sobre el uso de la lengua y otros contenidos) mediante el desarrollo de la competencia discursiva, aspectos ineludibles en un enfoque comunicativo (Martín Peris, 2010).

Para el contexto universitario, se han de seleccionar géneros del ámbito académico, según los rasgos tipológicos particulares como son el lenguaje formalizado, la escritura despersonalizada, el grado justo de cortesía y una máxima precisión (Perea Siller, 2013: 22). Serán estudiados secuencialmente, empezando por el discurso oral (exposiciones en el aula, debates y discusiones, etc.) y, seguidamente, el escrito. Pueden ser aquellos empleados en el aula (textos expositivos, descriptivos, ensayo, reseñas) y en la investigación, las comunicaciones, debates y otros (Vázquez, 2008). Los propios objetivos se establecen utilizando los criterios de evaluación, atendiendo a los niveles de competencia en fluidez, coherencia, corrección y alcance.

La tipología textual es extensa entre los géneros tradicionales, que se han ido tipificando a lo largo de los años, y entre otros que han surgido en los últimos tiempos, reflejo de la nueva realidad: WhatsApp, comentarios en blog, etc. Como hemos mencionado anteriormente, una de las características del género textual es su flexibilidad y actualización. Estos rasgos permiten su instrumentalización en la enseñanza desde el primer momento. El aprendiz puede deducir el tipo de texto e interpretar la información con pocos recursos y un bajo dominio, gracias al contexto en el que se desarrolla. No ocurre igual cuando un aprendiz ha de comprender unidades menores como la frase o sintagmas, o palabras sueltas fuera de contexto, justificando nuevamente la relevancia del género textual frente a otras unidades.

Todavía hoy el profesor no cuenta con muchas propuestas editoriales que trabajen desde el género textual aunque hay algunos ejemplos en Gente hoy, Aula, Abanico de Difusión. 
Es más común centrarse en la frase y la competencia gramatical, léxica y funcional (Vílchez Veleda, 2015: 3) como hace Método (Anaya). En este caso, presenta cada lección desde la frase y sus valores funcionales, para terminar con un tipo de texto.

\section{Secuenciación de las unidades. Aplicación para el aula}

Las unidades han de organizarse en torno a uno o dos tipos de género textual, adecuando el grado de dificultad de los objetivos al nivel de competencia en el que se trabaje. El tipo de texto ha de combinarse con varios aspectos: por un lado, con las tareas propuestas; por otro, con los contenidos del uso de la lengua (entre los que destacaremos la identificación y aplicación de los elementos discursivos) y otros contenidos no lingüísticos (Vílchez Veleda, 2015). Además, cada unidad ha de planificarse en un ciclo temporal que no ha de superar las 10 horas contabilizadas en la definición de Dolz (1994) según la cual la unidad didáctica es un ciclo desarrollado en el tiempo (entre 6-10 horas), con una finalidad concreta y articulado en secuencias temporales. En estas se especifican los objetivos en número limitado y seleccionados en relación al tema de la unidad. Para Dolz, los contenidos de lengua son el eje que estructura la secuenciación y que se examinan a lo largo de todo el proceso con evaluación inicial, formativa y sumativa (CE, 2001: 183). En este sentido, podríamos considerar el género textual como contenido de lengua, desgranado en los rasgos discursivos que lo definen.

A esta organización por unidades se ajustaría un enfoque por tareas, incorporando la instrucción de destrezas receptivas y productivas, orales y escritas, formales e informales, aunque se priorice una de ellas. El objetivo último de la instrucción es alcanzar un dominio discursivo adecuado al contexto y registro y para ello nuestra propuesta se basa en considerar las destrezas orales y escritas como instrumento procedimental y objetivo curricular.

Debido a este carácter procedimental de las destrezas, la posición temporal es fundamental. Las orales toman una posición inicial respecto a las escritas y con las primeras se introduce cada nueva unidad, por lo que se recomienda reservar este espacio inicial a las habilidades receptivas y productivas. No en vano, son habilidades presentes en todas las fases de aprendizaje y de las relaciones sociales: la comunicación entre profesor-alumnos y textos se realiza a través de la comunicación oral, tanto para trabajar las destrezas orales como las escritas, al igual que haría un usuario en la lengua materna. El elemento fónico es la materia prima de la oralidad y alrededor de este elemento sonoro se desarrolla el trabajo de aula: las receptivas, en las que habría que centrarse en las habilidades de comprensión auditiva, y las de creación o expresión oral (Moreno Fernández, 2002: 9-11).

A modo de ejemplo, exponemos una unidad de manera sucinta. Está ideada para un nivel B1 en una fase avanzada, destinado a estudiantes de un Máster de Olivicultura. En esta unidad se trabajaba el texto expositivo - explicativo, «envasado y etiquetado del aceite de oliva», dividido en dos bloques: exposición oral y exposición escrita. En el primer bloque, la primera fase se inicia con la visualización de un vídeo mudo sobre el envasado y etiquetado del aceite de oliva en https://www.youtube.com/watch?v=dPm5EG1bBos. Lo ven dos veces. Tras el primer pase, han de comentar de qué trata, señalar diferencias y semejanzas con el sistema de su país, etc. (expresión e interacción oral). Durante el segundo pase, deben fijarse y retener: 
-La historia, sabiendo que después resumirán el argumento.

-Las partes de la historia (estructura).

-Los objetos y maquinaria que aparecen.

-Anotar el léxico que no conocen para buscarlo más tarde.

Además, deben responder a algunas preguntas:

1. ¿De qué tipo de reportaje se trata (comercial, educativo, informativo, político, divulgativo, especializado...)?

2. ¿Cómo se trasmite la información? Estructura.

El objetivo es que vayan definiendo el texto expositivo. En este punto el profesor debe dirigir las preguntas para que el alumnado colabore en su elaboración y finalmente presentar una ficha que reúna los rasgos del texto expositivo-explicativo: finalidad (trasmitir información objetiva), papel del emisor (trasmisor, ha de evitar juicios de valor. Sin embargo, podemos encontrarlos de forma subliminal cuando se trata de textos comerciales, por ejemplo), género textual (presentación divulgativa o científica, reportaje, comunicación, textos jurídicos o humanísticos, entrada de una enciclopedia o diccionario, etc.), tipo de lenguaje (formal o informal, especializado o general), elementos discursivos (léxico, enlaces discursivos, etc.), estructura del discurso, etc. El profesor va añadiendo aquellos datos que los estudiantes necesitarán en la próxima tarea de la segunda fase: la exposición oral.

Depende del número de estudiantes, se formarán parejas o grupos de tres, aunque también puede ser individual. La tarea consiste en que ellos pongan texto al vídeo que han visualizado anteriormente. Han de planificar su exposición: acordar el destinatario (que determinará el registro), el objetivo (venta, educación, noticia-reportaje de algún canal televisivo, etc.) y ajustar la estructura al reportaje. Tras la exposición de cada grupo o cada alumno, según se haya organizado el curso, se revisan las pautas y características de este tipo de texto en su acepción oral. Seguidamente, se inicia el segundo bloque de la unidad: el texto escrito. Se plantean las diferencias y similitudes de ambos canales (este aspecto variará según en qué momento del curso se encuentre el grupo, pues probablemente ya sea algo que se ha visto en otra unidad anterior. Entonces, se refresca para reforzar la confianza).

Para esta fase de expresión escrita, es recomendable presentar distintos textos, de más fáciles o breves a más complejos o extensos. En esta ocasión pueden proponerse lo que sigue:

-Texto expositivo, breve e informativo http://www.sabor-artesano.com/etiquetadoenvasado-aceite.htm

http://www.juntadeandalucia.es/defensacompetencia/sites/all/themes/competencia/files/f ichas/pdf/1_Aceite_de_Oliva.pdf

-Texto expositivo, algo más largo y con explicaciones más extensas http://www.carapublica.es/normativa-sobre-el-etiquetado-del-aceite-de-oliva/

-Texto expositivo-explicativo, histórico y comercial, extenso. De este texto podrán sacar datos para la elaboración de la tarea escrita. http://www.mercasa.es/files/multimedios/1292927071_pag_066-085_Langreo.pdf

-Textos expositivos. Noticia http://www.diariocordoba.com/noticias/temadia/deoleoconvierte-cordoba-principal-envasador-aceite_1093933.html y de opinión en un blog 
http://anunciacarpio.blogspot.com.es/2013/08/guia-basica-para-comprar-seleccionarun.html . Servirán para entablar un debate (interacción oral)

De todo el material entregado o facilitado a los estudiantes se escogerá uno de ellos para trabajar en clase. En esta ocasión, seleccionamos uno sobre la normativa para envasado de aceites (se cita en los dos primeros epígrafes). Revisaremos la estructura, rasgos lingüísticos, conectores discursivos y tipo de lenguaje (neutro o personalizado, registro, etc.). Tarea final: Redactar un texto expositivo-explicativo sobre el envasado y etiquetado de aceite de oliva en su país o en España. Se le darán unas pautas de extensión, plazos o fechas de entrega, estructura, tipo de destinatario, objetivo, criterios de evaluación. Las tareas escritas se devuelven corregidas atendiendo como mínimo al cumplimiento de las tareas, la cohesión, corrección y alcance, como se señala en las escalas de calificación de SIELE (2017: 87 y ss.).

La oralidad genera confianza en la capacidad del estudiante sobre los objetivos marcados, es el medio natural que aporta autonomía en el aprendizaje de un estudiante-usuario como ocurre en la adquisición de una lengua materna (Kuhl, 2004) y lo prepara para avanzar, cambiar de canal, de soporte o de registros. La competencia discursiva de un usuario, más aún en ámbitos académicos, debe alcanzar textos orales y escritos (Swain, 1985), dos canales con entidad propia pero íntimamente ligados. Cada uno de estos canales se define por una serie de rasgos que justifican la separación que se suele hacer entre ellos y, al mismo tiempo, la inclusión de uno en otro (redes sociales), como se confirma en el propio aprendizaje donde el canal oral sirve de estrategia de comunicación en la comprensión y planificación de textos escritos y viceversa.

En resumen, es clave para unos buenos resultados enfocar tales cursos desde unas premisas básicas: enfoque comunicativo y por tareas tomando el género discursivo o textual como medida para trabajar la competencia discursiva. Se recomienda comenzar por las destrezas orales hasta concluir en las escritas, ya que este enfoque capacita al estudiante para enfrentarse a la labor creativa y científica que exige la actividad universitaria.

\section{Conclusiones}

El estudiante extranjero adscrito a programas de movilidad ha de integrarse en nuestras aulas como un alumno más de la comunidad universitaria pero generalmente lo hace en condiciones de inferioridad a causa de su bajo nivel de español, en especial respecto a la competencia académica. Con la pretensión de responder a esta situación se propone un plan de adecuación lingüística mediante cursos de apoyo que preparen al estudiante para participar y aprovechar su presencia en las clases regulares de su área de conocimiento.

Esta formación e instrucción puede ser más eficiente cuando se incluyen en un aprendizaje integrado (AICLE) dentro de una planificación consensuada por un profesorado que se involucre y se implique, extendiendo la enseñanza a todas las áreas de conocimiento, aportando un carácter trasversal a los contenidos de lengua (Gómez Camacho, 2013) que se materializan en el uso dado en las asignaturas universitarias.

A lo largo de dos cursos académicos hemos puesto en marcha un plan piloto para confirmar la efectividad de la propuesta. Se han organizado, por curso académico, dos grupos experimentales y tres de control. Todos los informantes se sometieron a las mismas pruebas de lengua. También tuvimos en cuenta sus propias apreciaciones y las de 
algunos profesores que fueron preguntados. Los grupos experimentales han dado resultados positivos tanto en el dominio de la lengua española como en el mayor aprovechamiento de su participación en la Universidad. Además, los resultados positivos de estas medidas se han corroborado por el cotejo con los resultados obtenidos por los participantes en los grupos de control, que han mantenido las mismas deficiencias que se venían detectando en años anteriores. Estos resultados nos reafirman en la necesidad de tomar medidas desde las mismas Universidades, ya que estas han de responder a los objetivos del EEES.

Este tipo de aprendizaje debe cumplir unos mínimos requisitos, como señalan algunos autores en sus distintas experiencias (Lasagabaster y Ruiz, 2010; Caballero y Reyes, 2011; Méndez y Pavón, 2012; Delicado y Pavón, 2015). Para todos ellos, cualquier puesta en marcha requiere un apoyo logístico de las instituciones competentes. Es igualmente importante la figura de un coordinador, que pone en contacto al profesorado de las distintas áreas, organiza la formación específica del profesorado y propone recursos y estrategias para actuar en el aula.

\section{Bibliografía:}

Bassols Puig, M. y Torrent BAdíA, A.M. (1997). Modelos textuales. Teoría y práctica. Barcelona: Octaedro.

Boletín OfiCIAL DE LA UnIVERSIDAD DE CÓRDOBA (2014). Acuerdo de Consejo de Gobierno, de 18 de diciembre de 2014, por el que se aprueba el Plan para el Fomento del Plurilingüismo de la Universidad de Córdoba (2014-2017). Recuperado de: http://www.uco.es/poling/pdf/Plan_Fomento_Plurilinguismo_UCO_14_17.pdf

CABallero Calavia, M y Reyes Fernández, M. (2011). «El nuevo alumno surgido del Plan de Fomento del Plurilingüismo de la Junta de Andalucía. España: estudio comparativo entre alumnos de $4^{\circ}$ de ESO». Revista Fuentes, 11, 139-160.

COMISIÓN EUROPEA (2008). Multilingüismo: una ventaja para Europa y un compromiso compartido [COM (2008) 566 final]. Recuperado de: http://eur-lex.europa.eu/legalcontent/ES/TXT/?uri=CELEX:52008DC0566

CONSEJO DE EUROPA (2001). Marco Común Europeo de referencia para las lenguas: aprendizaje, enseñanza, evaluación. Madrid: Instituto Cervantes-Ministerio de Educación Cultura y Deporte.

DelicAdo Puerto, G. y PAVÓN VÁzQuez, V. (2015). «La implantación de titulaciones bilingües en la Educación Superior. El caso de la formación didáctica del profesorado bilingüe de primaria en la Universidad de Extremadura». Educación y futuro, 32.

Dolz, J. (1994). «La interacción de las actividades orales y escritas en la enseñanza de la argumentación». CL \& E: Comunicación, lenguaje y educación, 23, 17-28.

FERNÁNDEZ LÓPEZ, S. (2011). «Nuevos desarrollos y propuestas curriculares. Programar a partir del MCER». Marco ELE, Suplementos 12, 3-64.

GómEZ CAMACHO, A. (2013). «El aprendizaje integrado de la lengua española y los contenidos de áreas no lingüísticas en los proyectos lingüísticos de centro». Porta Linguarum, 20, 103-115.

GutiérRez RiviLla, R. (2004). «Directrices del Consejo de Europa: El Marco Común Europeo de Referencia para las Lenguas: aprendizaje, enseñanza, evaluación (2002) », en Sánchez Lobato y Santos Gargallo (dir.), Vademécum para la formación de profesores. Enseñar español como segunda lengua (L2) / lengua extranjera (LE) (pp. 619-641). Madrid: SGEL. 
Instituto Cervantes (2013). Diploma de español. Guía de examen. Recuperado de: https://examenes.cervantes.es/

INSTITUTO CERVANTES (2017). Guía oficial para preparadores del SIELE. Recuperado de: https://siele.org/web/guest/landing-profesores-libre

KuHL, P. (2004). Early Language Acquisition: Cracking the Speech Code. Seatle: University of Washington.

LASAgabaster, D. y Ruiz De ZARobe, Y. (ed) (2010). CLIL in Spain: implementation, Results and Teacher Training. Cambridge: Cambridge Scholars Publishing.

LORENZO Berguillos, F. (2013). "Genre-based curricula: multilingual academic literacy». Content and language integrated learning, International Journal of Bilingual Education and Bilingualism, 16:3, 375-388. DOI: http://dx.doi.org/10.1080/13670050.2013.777391

MARSh, D., PAVÓN VÁZQUEZ, V. y FRIGOLS MARTÍN, M. (2013). The higher education language landscape. Insuring quality in English Language Degree Programmes. Valencia: Valencian International University.

MARTín PERIS, E. (2010). «Perspectivas actuales sobre la metodología de la enseñanza», Ponencia en Congresos internacionales de la lengua española. Recuperado de:

$\mathrm{http}: / /$ congresosdelalengua.es/valparaiso/ponencias/lengua_educacion/peris_ernesto_m.htm

Martínez-Atienza, M. y Perea Siller, J. (2015). «Los géneros académicos en el Grado de Filología Hispánica». Linred, febrero de 2015.

MÉNDEZ GARCÍA, Ma y PAVÓN VÁZQUEZ, V. (2012). «Investigating the coexistence of the mother tongue and the foreign language through teacher collaboration in CLIL contexts: perceptions and practice of the teachers involved in the plurilingual programme in Andalusia». Intenational Journal of Bilingual Education and Bilingualim. 15: 5 (pp. 573-592). London: Routledge.

MORENO FERNÁNDEZ, F. (2002). Producción, expresión e interacción oral. Madrid: Arco Libros.

PAStor Cesteros, S. (2014). «Aprendizaje de contenidos a través del español como segunda lengua en la educación superior: un puente entre la lengua y el conocimiento». Euro American Journal of Applied Linguistics and Languages E-JournALL, Vol. 1, Issuie 1, 15-30.

PAVÓN VÁZQUEZ, V. (2006). «Análisis de las causas por las que la enseñanza de las destrezas orales obtiene resultados insatisfactorios en el entorno del aula y propuestas de mejora». En Amengual, Garau y Salazar (editoras), Adquisición y aprendizaje de lenguas en contextos plurilingües. Ensayos y propuestas aplicadas (pp. 417-431). Baleares: Universitat les Illes Balears- AESLA.

PAVÓN VÁzQUEZ, V. (2014). «Enhancing the quality of CLIL: Making the best of the collaboration between language teachers and content teachers». Encuentro, 23, 115-127.

Perea Siller, J. (Coord.) (2013). Comunicar en la universidad. Descripción y metodología de los géneros académicos. Córdoba: Universidad de Córdoba.

SWAIN, M. (1985). «Communicative competence: some roles of comprenhensible input and comprenhensible output in its development». En Gass y Madden (eds.), Input in second language acquisition. Rowley- Massachussets: Newbury House Publishers.

TRUjILlo SÁEZ, F. (2010). «La competencia en comunicación lingüística como Proyecto». Lenguaje y textos, 32, 35-40. 
VÁZQUEZ, G. (2008). «¿Qué español enseñar? Preguntas frecuentes», I Congreso Internacional de enseñanza e investigación en ELSE. Argentina. Recuperado de: http://www.lenguas.unc.edu.ar/elsecongreso/teleconferencia_vazquez.pdf

VílCHEZ VelEDA, A. (2015). «La anécdota: un estudio contrastivo alemán-español». RedELE, 16. Recuperado de: http://www.mecd.gob.es/redele/Biblioteca-Virtual/2015/memoriasmaster/Alberto-Vilchez.html 